\title{
Digital Image Display Station Performance Requirements Based on Physician Experience With a Prototype System
}

\author{
Paul Fisher, Brent Grover, Gerhard Brauer, and Gordon Ritchie
}

\begin{abstract}
The authors report on observations of and interviews with physicians using a prototype digital image display and reporting station. While the users generally agree that image quality is clinically satisfactory. they are unanimous in their opinion that improvements in the man-machine interface are required before case review by this mechanism is clinically acceptable in a production environment. A model image and information user interface is presented. It was developed in answer to the needs of radiologists and referring physicians operating in the imaging department of a community acute-care facility. In such an environment images and related information must be communicated quickly and often simultaneously to different parts of the department and hospital. The user interface with the management system and the management system itself must address the varied functions and the needs of both the medical and clerical staff. Image enhancement processes, for example, must be restricted to those that quickly provide significantly more perceivable diagnostic information. Little-used processes that may occupy significant portions of the display and the console's computing power must be trimmed or eliminated.
\end{abstract}

(C) 1989 by W.B. Saunders Company.

D IGITAL medical imaging subsumes those processes that result in digital image files for display, in the first instance, on video monitors. They include computed tomography (CT), nuclear medicine (NM), ultrasonography (US), nuclear magnetic resonance imaging (MRI), positron emission tomography (PET and SPECT), and digital subtraction angiography (DSA); they bear about $40 \%$ of a modern medical imaging department's routine case load. Images from radiographic and most fluorographic examinations that account for the remaining $60 \%$ of the department's work are recorded on $\mathrm{x}$-ray-sensitive film.

Great effort has gone into converting the

From the School of Health Information Science, The University of Victoria, British Columbia; and Medical Imaging, Victoria General Hospital, British Columbia, Canada.

Address reprint requests to Paul Fisher, PhD, School of Health Information Science, The University of Victoria,

Victoria, BC, Canada V8W $2 Y 2$.

(C) 1989 by W.B. Saunders Company.

0897-1889/89/0203-0009\$03.00/0 outputs of radiography and fluorography to digital format ${ }^{1}$ so that images from all modalities can be transmitted over a common image network. Conversion of all image outputs to digital is, however, only one of the tasks required to achieve a computer-managed medical imaging system. Image acquisition is essentially proven technology. The question remaining is what level of digital technology is needed to provide the fidelity required to support image-based diagnoses.

The development of an effective picture archive and communication system (PACS) is the logical next step, and is at least as important as image fidelity to the success of digital imaging. The performance of the PACS, as perceived by the user at the work station, will largely determine the success of digital imaging in medical diagnosis. That the digital images and information must be organized in a fashion similar to what physicians are accustomed to in film-based systems has been acknowledged..$^{2-4}$ Research is now required to determine (a) which information distribution topology most effectively and economically provides the information to the point of use: the diagnostic work station, and (b) which image processing and management functions are most likely to enhance the diagnostic process.

\section{DIGITAL IMAGE ACQUISITION}

Of the various methods developed to acquire digital radiographic and fluorographic images, three have persisted and will become the norm for digital radiography. The first and simplest is the digitizing of conventional radiographic films using an optical scanner. ${ }^{5}$ This method has been found to be clinically satisfactory, but the continued use of film and related processes and materials means that the capital cost of digitizing and display equipment is added of that of the existing film-handling system. However, as long as conventionally acquired $\mathrm{x}$-ray films remain in use, the film digitizer (scanner) will play an important role in the medical imaging department. The second method is the digitizing of the video signal 
from an image intensifier. ${ }^{*}$ Image intensifiers are already used for fluoroscopic studies and their use has been extended to radiographic studies of the chest and peripheral anatomy. ${ }^{1,6,7}$ The latter has been made possible by the development of image intensifiers large enough to accommodate the whole human chest. ${ }^{8}$ However, this method has a drawback in that a large image intensifier is not as versatile as a film cassette and cannot, for example, be slipped under a recumbent patient. The use of reusable memory screens, the third method, circumvents this problem by combining the desirable features of both the film cassette and digital technologies. This method is the one most likely to receive initial support from imaging department staffers simply because it requires the least change in the patient-handling routine.

\section{IMAGE VIEWING}

The development of the software and hardware needed for the display and reporting of digital images is becoming, for psychological and conceptual reasons, a very challenging task. While difficulties will ease as medical staff becomes increasingly comfortable with computers, the initial acceptance of digital imaging technologies will be facilitated if little change in the users' work habits is required. In radiology, these work habits center on the image-viewing area. Several aspects must therefore be considered in developing a digital image viewing station.

\section{Ergonomics}

As in any work station, physical design must be comfortable for the user and appropriate to the task. In the authors' experiences, physicians are reluctant or unwilling to use keyboards. Logical menus accessed with a pointing device (eg, mouse, joystick, light pen, etc) are the appropriate mechanism for a physician doing routine clinical work at a digital imaging work station. Pointing to and marking image features are also best achieved by using such a device. It

\footnotetext{
*An image intensifier has an $x$-ray-stimulable phosphor screen for input. Emitted photons from the back side of the input screen are focused, using an electromagnetic lens, onto a smaller output phosphor which scintillates in the visible region, revealing the image to a video camera.
}

must be comfortable to handle and operate in a manner logically and visually acceptable to the user. The number, size, placement, and orientation of the monitors also requires careful consideration.

\section{Psychophysical}

Factors falling into this category are often overlooked. For example, while digitization to a lower level of spatial resolution reduces the information content of an image, the actual size of the image presented will affect how much of that information is perceptible. In radiography and fluorography, images are customarily about life-sized. Given the positive correlation between image size and signal detection performance, ${ }^{9}$ video images should also be presented as near life-size as possible to optimize the amount of diagnostic information perceivable to the reader at any given level of spatial resolution.

Radiographic films are usually presented on light boxes in areas with low or no ambient light and with nondiagnostic areas of the film darker than the anatomical structures being examined. These two factors combine to reduce distracting visual "noise" and to dilate the pupils so as to increase the amount of light reaching the retinas, thereby enhancing discrimination of low-contrast objects. In spite of this, the default presentation format of many digital image work stations has dark anatomical structures on a light background, ie, the reverse of that favored by North American radiologists.

\section{Informatics}

The way in which a user accesses the clinical image and information databases will affect the clinical utility of digital imaging systems. The configuration of the databases and the management application must take into account that the end result of a radiographic examination is the diagnosis made by the radiologist, and not the image(s) on which it is based. This is often not reflected in many existing digital medical image database applications that include fields to identify the image but few or no provisions for extensive clinical reporting or administrative record keeping. Additional database applications are often required for this purpose. A complex and often costly interface between the two data- 
base applications is needed in order to make the system clinically useful.

A record of patient images is necessary, but the diagnosis is key to patient management. Though medical images without the associated diagnostic or therapeutic information are of little clinical use, clinical information is extensively used without the images: eg, for report entry, patient records, statistics, billing etc. The single diagnostic database application should, accordingly, first address patient information files through which the patient's images can subse quently be accessed (Fig 1). Since the diagnostic functions are the only ones that require access to the image display function, it makes sense to maintain two separate databases, one for images and the other for information that can be accessed independently by a number of nondiagnostic functions and simultaneously by the diagnostic ones (Fig 1). Disencumbered of large image data blocks, records in the smaller information database could be more quickly accessed by the more frequently used administrative and clerical functions without interfering with the image display function.

The clinical user-machine interface must be designed to take into account the variety of functions performed by the clinical and technical staff in the department. A single interface for all of these functions forces each type of user to waste time selecting those menu items specific to his or her function. The following list of basic

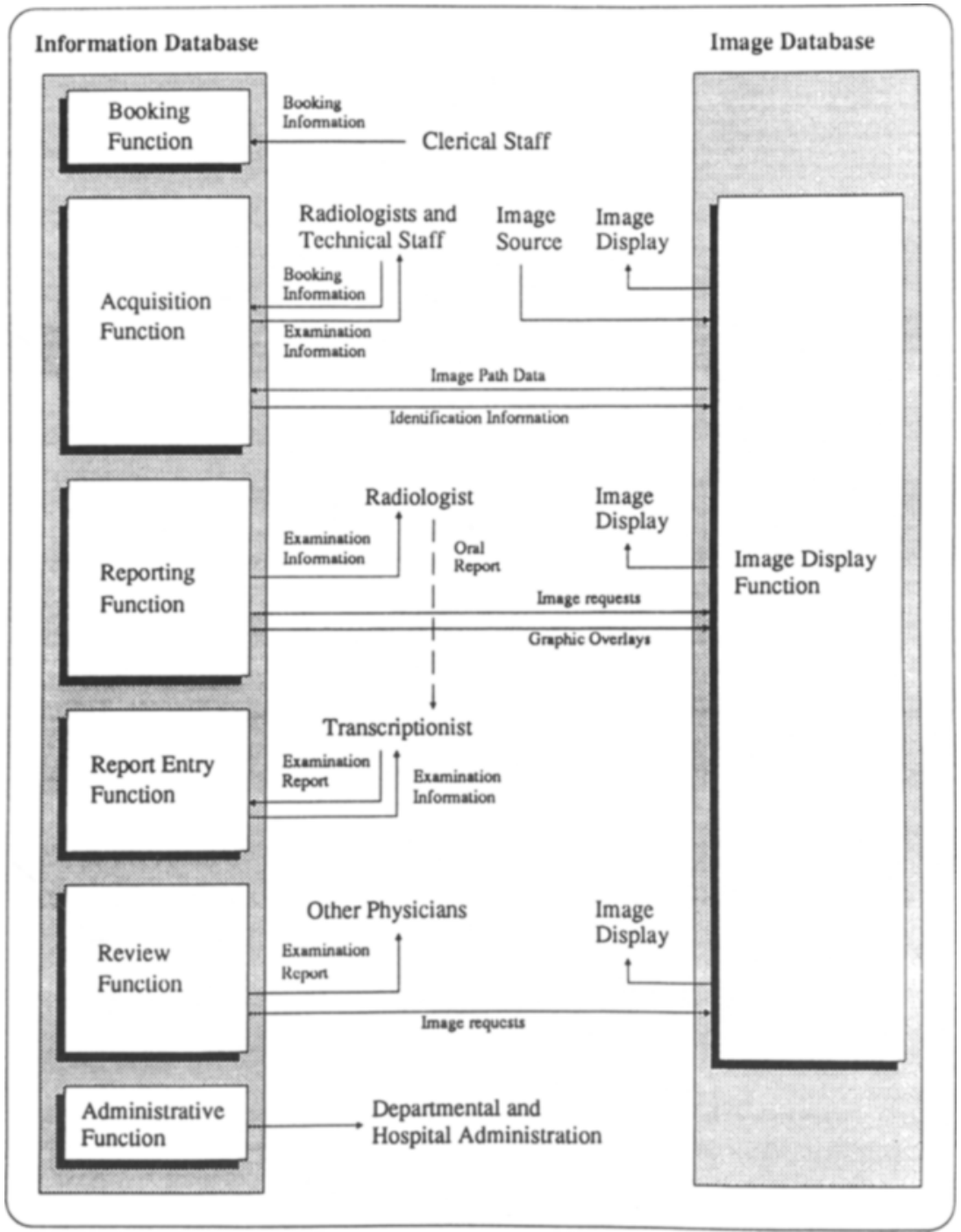

Fig 1. Conceptual topology for a diagnostic database application for a medical digital imaging system. The application addresses two databases but only the information database can be directly accessed by users. 
functions, also shown in Fig 1 , represents a possible activity sequence for each.

Booking. The user is presented with a blank request for examination (RFE), a record in the information database, into which patient demographics and clinical data are entered. A separate window shows the user a schedule of the dates and times available for that type of examination.

Acquisition. The radiographer selects a case from the list (by patient name) of pending examinations for a particular room. The RFE, as completed at booking, is presented and, simultaneously, a record is opened in the image database to accept new images for that examination. When the procedure is complete, an updated list of pending examinations is presented.

Reporting. The radiologist selects from a list (by patient name) of completed but unreported examinations for a particular modality. The respective $\mathrm{RFE}$ is presented and, simultaneously, images for that examination are presented on the image screens. A window on the text screen lists all previous examinations on record for that patient. Those available for viewing immediately are indicated.
Report transcription. The transcriptionist selects from a list of completed and reported examinations for which transcription from voice is required. The incomplete RFE is presented and the user enters the report.

Review. The physician enters the name of a patient whose case is to be reviewed, and is presented with a list of examinations for that patient. The examination of interest is selected and the RFE and related images are presented at the workstation. Images from related examinations may be requested from a list contained in a separate window.

The time taken to perform each function, especially the diagnostic ones, is critical to the efficient operation of the department and is most likely to immediately affect the acceptability of a digital-imaging system. Table 1 presents a comparison of the times taken to perform the various functions in the conventional film-based system with those for the digital imaging system the authors are familiar with. Users of that system are unanimous in their opinion that the current system is too slow to accommodate the daily case load of the department. Though increases in the time required to perform some of these functions

Table 1. A Comparison of Times Required to Perform Various Functions in the Conventional and Digital Imaging Systems

\begin{tabular}{|c|c|c|c|c|c|}
\hline \multicolumn{3}{|c|}{ Conventional Film System } & \multicolumn{3}{|c|}{ Digital Imaging System } \\
\hline & & $\begin{array}{l}\text { Elapsed } \\
\text { Time (Sec) }\end{array}$ & & & $\begin{array}{l}\text { Elapsed } \\
\text { Time (Sec) }\end{array}$ \\
\hline Image acquisition & & & Image acquisition & & \\
\hline Patient verification & 10 & - & Patient verification & 10 & - \\
\hline Patient exposure* & - & - & Patient data entry & 90 & - \\
\hline Subtotal & 10 & 10 & Patient exposure * & - & - \\
\hline Image preparation & & & Subtotal & 100 & 100 \\
\hline Film handling & 30 & - & Image communication & & \\
\hline Film processing & 120 & - & Transfer to file $\dagger$ & - & - \\
\hline Subtotal & 150 & 150 & Update database $\dagger$ & - & - \\
\hline Image communication & & & Transfer to console $\dagger$ & - & - \\
\hline Mount film & 30 & - & Subtotal & 231 & 331 \\
\hline Log location & 30 & - & Image display & & \\
\hline Subtotal & 60 & 210 & Sign on & 25 & - \\
\hline Image display & & & Select patient & 7 & - \\
\hline Locate film & 5 & - & Display four images & 52 & - \\
\hline Display film & 7 & - & Adjust presentation $\ddagger$ & 58 & - \\
\hline Magnification & 15 & - & Exit & 7 & - \\
\hline Exit & 3 & - & Subtotal & 230 & 561 \\
\hline Subtotal & 30 & 240 & & & \\
\hline
\end{tabular}

Figures are based on the acquisition and display of four wrist images.

- No times indicated since these are very variable and not part of the imaging system per se. However, while in conventional radiographic procedures, the room is available for the next examination. Immediately after exposing the film in the digital system, no further images can be acquired until all current ones have been transferred to image files.

† Many of the processes in image communication occur in parallel and cannot be separated in time.

fimages must be manipulated to the proper orientation, polarity, and window before diagnostic viewing can commence. 
may be tolerable, additional overhead in the viewing function is not.

\section{Image Processing}

While the actual information content of radiographic film images is significantly higher than that of most digital images, $\dagger$ perception of that information is restricted by the static nature of the film presentation format and the psychophysical limitations of the human visual system. ${ }^{9}$ Digital-image display stations allow the user to transcend the above limitations by providing image processing functions. Windowing, enlargement and zooming, edge detection, and histogram equalization are among the image-enhancement processes that have been successfully applied to clinical images from a variety of modalities. ${ }^{10,11}$ These processes can, and should, be applied to some degree prior to initial (diagnostic) viewing, ie, as image preprocessing.

While significant work has been done in the area of processing radiographic images to facilitate the diagnosis, ${ }^{12}$ only a small number of such processes are in routine clinical use. Of these, most are applied in image modalities that rely on computers for image generation, eg, MRI, CT, DSA, etc. ${ }^{10}$ Processes will continue to be identified as useful for the variety of diagnostic imaging procedures but, for each process the following factors must be considered before clinical implementation: Do the features enhanced by the process contribute positively to the diagnosis? Are some diagnostic features eliminated in a process that enhances others? To images of which modalities, procedures, pathologies, and anatomical regions can a particular process be successfully applied? From which processes does a particular user derive the most clinical benefit? $\$$

The diagnostic application in the digital imaging system must identify the clinical query, anatomical region, modality, and user, so as to provide the current user with only those processing options applicable to the case. Inappropriate

$\dagger$ The highest spatial-resolution digital images in clinical use are $1.024 \times 1,024$. To achieve resolution equivalence with radiographic film, images would have to be digitized to at least 4,096 × 4,096.

$\$$ The operative word here is "clinical." Recent work by the authors reports that physicians performed well clinically with images they considered inferior in quality. and clinically useless image processing discourages the use of diagnostic imaging workstations by overwhelming the user and by occupying processing power, thereby reducing the apparent speed of the workstation. In addition, it clutters up the application screen with unnecessary menu items.

A common complaint from users of diagnostic work stations is that, unlike film, the initial image presentation is inappropriate and precious time must be used to make the image presentable. To provide an immediately presentable image together with a simple menu of appropriate additional processes, image preprocessing based on modality, pathology, anatomy, and user will be an important step in providing an acceptable digital-imaging viewing system.

\section{IMAGE COMMUNICATION: PACS}

The currently popular PACS network concept is one in which images from all modalities are accessible at a number of generic work stations (Fig 2). Implementing this concept today will be costly and, in the authors' opinion, will not meet the demands of a full-service medical imaging department, given current technology. Furthermore, making all images continuously available at all work stations is an unnecessary and costly luxury that does not reflect current film-usage patterns. Now, most cases of a single type are reported at the same multiviewer (the film equivalent of the work station). The incidence of direct reference to or comparison with images from other examinations is very low. ${ }^{13}$ Work stations
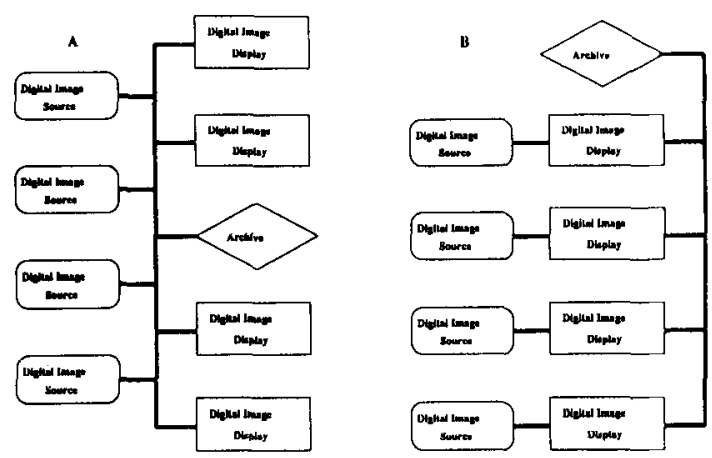

Fig 2. Alternative network topologies for PACS. (A) All images transmitted over a common network and all workstations are truly multimodal. Routine network traffic is high. (B) Each image source has a dedicated workstation that is only indirectly multimodality but routine network traffic is low. B conforms more closely to film image traffic patterns. 
should therefore be dedicated to examinations from a particular modality and physically configured to suit those particular examinations. Fluorographic and tomographic examinations, for example, require the simultaneous display of a large number of high-resolution images and therefore would need the greatest number of viewers, while images from a nuclear-medicine examination are generally of low resolution and are few in number. Clearly, installing the same type of work station in both areas would not be cost effective.

If most images in one work station were to come from one modality, the acqusition devices generating those images would not need to share a common communication network but rather would be directly connected to the processor dedicated to the workstation for that modality (Fig 2). Only subsequently, during off-peak clinical hours, would all images be sent over a common network to a file server for archiving purposes. This concept allows a relatively trafficfree network to exist for the infrequent communi- cation of images between modalities and for the retrieval of images from the archive.

\section{CONCLUSION}

To be accepted readily, digital medical imaging, communication, and management must be based on current usage patterns of film images in a routine clinical environment. It is probably counterproductive to suggest to users of the existing film system that they should change work patterns that have evolved over decades and work. Also, attention must be paid to the image traffic patterns and to patterns of information use at the clinical level to achieve a system that is dynamically acceptable to the medical user.

Basing the development of a digital image communication system on present film image usage

first step. Just as the manual system developed around film image use, a system of digital-image handling will evolve over time as its unique features are appreciated and effectively exploited by the medical community.

\section{REFERENCES}

1. Goodman LR, Wilson CR, Foley WD: Digital radiography of the chest: Promises and problems. A J Radiol 150:1241-1252, 1988

2. McNeill KM, Seeley GW, Maloney K, et al: Comparison of digital workstations and conventional reading for evaluation of user interfaces in digital radiology. Proc SPIE 914B:872-876, 1988

3. McNeill KM, Seeley GW, Maloney K, et al: Comparison of digital workstation and a film alternator. Proc SPIE 914B:929-932, 1988

4. O'Malley KG, Giunta JA: The alternator: Determination of its fundamental features, as a basis for design of a PACS diagnostic workstation. Proc SPIE 914B:988-994, 1988

5. Schwenker RP, Edgar H: Film-screen digital radiography. Radiography 51:233-235, 1985

6. Brauer GW, Fisher PD, Hanley JA, et al: Signal detection in digital chest-phantom images acquired with an image intensifier. J Digital Imaging 2:42-47, 1989

7. MacMahon CH, Metz CE, Doi K, Kim T. Giger ML and han HP: Digital chest radiography: Effect on diagnostic accuracy of hard copy, conventional video, and reversed gray scale video display formats," Radiology, Vol. 168, pp. 669673,1988

8. Kheddache S, Mansson LG, Schlossman D, et al: Digital chest radiography with a large-screen image intensifier. Electromedica 55:21-27 1987

9. Fisher PD, Brauer GW: Impact of image size on effectiveness of digital imaging systems. J Digital Imaging 2:1-4, 1989

10. Buchmann F, Coumans J, Dallas W, et al: Digital image processing. Medicamundi 30:37-45, 1985

11. Scherrier RH, Johnson GA: Regionally adaptive histogram equalization of the chest. IEEE Trans Med Imaging MI-6:1-7, 1987

12. See Proc SPIE 914A: 1988, for a great variety of recent papers on this subject

13. Fisher P, Brauer $G$, Nosil J, et al: Image storage requirements for PACS predicted from current film image traffic in a 12 room imaging department. Presented at the 51st Annual Meeting of the Canadian Association of Radiologists, Edmonton, Alberta, Canada, June 12-16, 1988 